\title{
A Review on Transposons and its Utilization as Genetic Tool
}

\author{
Sadia Perween, Deepak Kumar* and Anand Kumar \\ Department of Plant Breeding and Genetics, Bihar Agricultural University, Sabour \\ (Bhagalpur), India \\ *Corresponding author
}

\begin{tabular}{|c|}
\hline $\begin{array}{l}\text { K e y w o r d s } \\
\text { transposons, } \\
\text { insertational } \\
\text { mutagenesis, } \\
\text { transposon tagging }\end{array}$ \\
\hline Article Info \\
\hline $\begin{array}{l}\text { Accepted: } \\
15 \text { January } 2020 \\
\text { Available Online: } \\
10 \text { February } 2020\end{array}$ \\
\hline
\end{tabular}

\section{A B S T R A C T}

Transposable elements, found in almost all organisms, are short sequences of DNA that have the ability to move from one location to the other locations in the genome. Transposons make up $10 \%$ of eukaryotic genomes. They provide a means for genomic change and variation, particularly in response to stress (McClintock's stress hypothesis). There is no known example of transposon playing a normal role in development. They are called as selfish DNA and can be used as genetic markers, as mutagens for transposon tagging and isolation of gene, as transformation vectors and as cloning vehicle. Transposons are potent forces of genetic change and have a significant aspect in the evolution of many genomes. DNA transposons can be used as genetic tools to introduce a piece of foreign DNA into any genome. They have been utilized for transgenesis and insertional mutagenesis in diverse organisms, as these elements are not generally dependent on host factors to facilitate their mobility. Thus, DNA transposons are powerful tools to analyze the regulatory genome, study the development of embryo, identify genes and pathways involved in disease or pathogenesis of pathogens, and helps in gene therapy. Transposons are used for gene cloning since insertion of a transposon into a gene upsets its function which develops a visible mutant phenotype. When the DNA sequence of the transposon is known, it is possible to clone the disrupted gene by employing the transposable element as a tag to locate the segment of DNA possessing the element. Transposon tagging involves initiating transposition, screening for mutations caused by transposon insertion, discovering the element responsible for the developed mutation, and cloning the tagged gene.

\section{Introduction}

Transposable elements (jumping genes, insertion sequences or mobile DNA elements) are short sequences of DNA that can move from one location to the other in the genome, often making duplicate copies of them in the process. They are found in almost all organisms (both prokaryotes and eukaryotes), typically in large numbers viz., they make up approximately $50 \%$ of the human genome and up to $90 \%$ of the maize genome (SanMiguel, 1996).

\section{Types of transposons}

There are the transposable elements that need reverse transcription (i.e., the transcription of 
RNA into DNA) to transpose, called retrotransposons or class 1 TEs, whereas, some elements do not have such requirement for transposition and are known as DNA transposons or class 2 TEs.

\section{DNA transposons}

The complete or "autonomous" class 2 TEs encodes the protein transposase, required for insertion and excision. A few of these TEs also encode other proteins. Its mobility is always on its own (insertion or excision), from the genome by means of "cut and paste" mechanism.

\section{Retro transposons}

Class 2 elements, known as retrotransposons, move by the action of RNA intermediaries. i.e. they do not encode transposase; rather, they produce RNA transcript followed by action of reverse transcriptase enzymes to reverse transcribe the RNA sequences back into DNA, which is to be inserted into the target site.

\section{Autonomous and nonautonomous transposons}

The class 1 and class 2 TEs can be either autonomous or non autonomous. Autonomous TEs have their own mobility, while non autonomous elements require the presence of other TEs to move from one place to another in genome. The reason behind it is that non autonomous elements lack the gene for the transposase or reverse transcriptase that is required for their transposition, so they need these proteins from another element in order to move along the genome.

For example $A c$ elements are autonomous and can move on their own, whereas, $D s$ elements are non autonomous and require the presence of $A c$ in order to transpose.

\section{Conservative and replicative transposition}

In conservative transposition, the sequence is excised from its original position and reinserted elsewhere without increasing its copy number, whereas, in replicative transposition transposons creates a copy of itself and the copy is inserted at a new position.

\section{Bacterial transposons}

Most transposons in bacteria transpose as DNA. On the other hand, in eukaryotes they are mostly retrotransposons. Short direct repeats are created at the site of insertion flanking the mobile element.

1. IS Element: it constitutes inverted repeat at each end of insertion sequence and between the inverted repeats is a protein coding region encoding enzymes required for transposition. Short direct repeats are adjacent to both ends of the inserted element.

2. Composite transposons: they are larger than IS elements containing protein coding region along with those required for transposition and usually carry genes for antibiotic resistance.

3. Non composite transposons: they terminate with IS element but contain terminal inverted repeats.

\section{Transposable elements in eukaryotes}

Transposons constitute $10 \%$ of eukaryotic genomes being similar in structure to bacterial IS elements with retroransposons being much more profuse.

\section{Element 1 - Yeast ty elements:}

The yeast has approximately 35 copies of a transposable element called as $\mathrm{Ty}$ in its haploid genome. 


\section{Element 2 - Drosophila transposons:}

Drosophila transposons consists of retrovirus like elements i.e. retro transposons and are 5000 to 15,000 nucleotide pair long.

\section{Element 3 - Controlling elements in maize: Mcclintock's experiments: the ds element:}

In the 1950s, Barbara McClintock studying corn kernels found that, rather than being purple or white; they had spots of purple pigment on normally white kernels. The genetic and cytological studies concluded that the spotted phenotype was not the result of any conventional kind of mutation but due to a controlling element, which is now known as a transposon.

The spotted kernelis the result of wild type $\mathrm{C}$ and $\mathrm{c}$ (colorless) gene. The presence of $\mathrm{C}$ gene makes the kernel purple, c (colorless) mutations block purple pigment production, so the kernel is colorless. During kernel development, mutation reverts, due to which there is a spot of purple pigment. The genetic nature of the reversion is approved by the fact that descendants of the cell which had the reversion also can give rise to pigmentation. The sooner in development the reversion occurs, the larger is the purple spot. McClintock determined that the original $c$ (colorless) mutation is the resultant of a mobile controlling element, a genetic factor known as Ds (Dissociation), which gets inserted into the $\mathrm{C}$ gene. The action of Dsrelies on the presence of an unlinked gene, Ac (Activator).

Ac is needed for transposition of Ds into the gene. It can also move the Ds out of the $\mathrm{C}$ gene, giving rise to the wild type revertant, i.e., a purple spot. Ac is the autonomous element, and therefore, mutations caused by Ac are unstable. In contrast, Ds is the nonautonomous element of the family. The Ds mutations are stable only if Ds are present; whereas unstable in the presence of an Ac element.

Ds is immobile in the absence of Ac and remains as a stable insertion in the chromosome. In the presence of an Ac element, it gets activated, causing it to transpose to a new site or to break the chromosome in which it is located.

\section{Biological significance of transposons}

Transposons are a means for genomic change and variation, especially in response to stress (McClintock's stress hypothesis). There is no known example of an element playing a normal role in development, hence called as selfish DNA.

\section{Application of transposons}

Transposons as genetic markers (Izsvak and Ivics, 2004)- as it changes the pattern of restriction fragment analysis

Transposons as mutagens and transposon tagging (Whitham et al., 1994 Dinesh et al., 1995) for isolation of gene

Transposons as transformation vectors

Transposons as cloning vehicle

Transposons are powerful forces of genetic change and have a significant role in the evolution of many genomes. As genetic tools, DNA transposons can be utilized to insert a piece of foreign DNA into a genome. They have been aided for transgenesis and insertional mutagenesis inorganisms, as these elements are not generally dependent on host factors for their mobility. Thus, DNA transposons are useful devises for analyzing the regulatory genome, study embryonic development, identifying genes and pathways related to disease or pathogenesis of pathogens, and contributing to gene therapy. 


\section{Transposon tagging}

Transposons are used for gene cloning as insertion of a transposon into a gene disarms its function, producing an observable mutant phenotype. When the DNA sequence of the transposon is known, it is likely to clone the disrupted gene by using the transposable element as a tag to identify the segment of DNA containing the element. Transposon tagging includes inducing transposition, screening for mutations caused by insertion, recognizing the element responsible for mutation, and finally cloning the tagged gene. Transposons from one organism sometimes also transpose in heterologous systems. Therefore it is possible to use transposontagging method to clone genes in different organisms, inclusive of those where there are few other methods available for gene isolation.

\section{Characteristics of transposons relevant to tagging}

Class I transposons are used for tagging in mammals and yeast, whereas; class II transposons are most frequently used in tagging schemes for bacteria and plants.

\section{Regulation of Transposition}

It is essential to have a few methods for controlling the frequency of transposition. There are families of transposable elements containing non autonomous controlling elements that cannot produce transposase but are mobile in the company of the transposase produced by an "autonomous" family member. Similarly, two-element system is mostly employed in transposon-tagging techniques where a stable transposase source (e.g., a transposon immobile due to deletion of one of its direct repeats) is employed to move nonautonomous elements. This system has the edge as once new insertions have been created by merging the two types of elements, the transposase source can be segregated. As a result, the new insertions are stabilized and thus any mutant phenotypes generated gets stabilized. Transposase can be reintroduced later for remobilizing the element to reverse the mutant phenotype or to produce new alleles of the tagged gene.

\section{Obtaining the Sequence of the transposon tag}

Transposon as a molecular tag for gene cloning needs to have a known DNA sequence. Many transposons have been well defined and characterized. New transposons can be identified by mobilizing transposition followed by identifying transposon insertions into known genes (generally by recognizing unstable alleles of the gene). The mutant allele is sequenced to determine the sequence of the inserted transposon.

\section{Integration site preference}

The efficiency of transposon tagging at any gene is decided by a number of factors. For a transposon to be perfect for tagging purpose, there would be no preference for the integration site in the host genome, but many transposons show some type of site preference. The examples of preferences include $C$. elegans Tc1 element having a TA dinucleotide target site(Rozenzweig et al., 1983a and Liao et al., 1983) and Drosophila P element preferentially inserting at the 5 '-ends of transcription units(Kidwell, 1994).

\section{Transposition to linked sites}

A characteristic of many transposons is their ability to transpose to linked sites making it useful for some types of screens, but in the process makes random transposon mutagenesis more strenuous. In some cases, methods have been studied to identify 
transposition to unlinked sites. An Arabidopsis Ds element harboring a positive selectable marker was linked to IAAH (a marker that can be selected against). The Ds element was mobilized, by selecting for the Ds marker and selecting against IAAH, lines were discovered in which the transposon had jumped to a position unlinked to IAAH.

\section{Endogenous versus heterologous transposons}

Another feature in transposon-tagging experiments is to use either endogenous unmarked transposons, which are plentiful in some backgrounds, or to construct transposons specifically for transposon tagging and to allow movement of a single transposon or a few transposons per genome.

The greater the number of mobile transposons and the higher the transposition frequency, the greater is the chance of tagging any particular gene. A large number of background transposons create difficulty in the linkage studies necessary for identifying the particular transposon inserted into the gene of interest. High rates of transposition result in unstable mutations, creating difficulty in linking a transposon with a mutant phenotype.

The application of only one or a few transposons per genome facilitates identifying the transposon inserted into the gene of interest and thus accelerates cloning of the gene. More individuals must be screened in order to get insertions into the gene of interest.

Heterologous two-element, transposontagging system can be used to ensure the mobilization of only a few elements in the genome in the presence of the transposase, allowing removal of the transposase by segregation to prevent further transposition.

\section{Transposon and transposase engineering}

Transposons are designed to transport selectable markers for carrying part of a plasmid that can be selected for in E. coli, facilitating clone development of flanking DNA by plasmid rescue technique. A marker linked to the transposase source allows removal of the transposase in future by segregation from the tagged gene.

\section{Transposon tagging strategies: obtaining insertions into genes}

\section{Random mutagenesis}

In random mutagenesis, transposons are mobilized to produce a library of entities with diverse transposon insertions followed by screening for mutant phenotypes of interest from the developed library. In a two-element system, transposons are moved by positioning stable transposase into the background of the non autonomous transposons. Dominant detectable mutations are spotted in the M1 generation, and recessive detectable mutations are seen segregating in the M2 progeny. Individuals having a transposed element are termed "transposants".

\section{Directed mutagenesis}

Directed tagging schemes are of two types. In both cases, the target gene has been identified by its mutant phenotype.In the first type of directed mutagenesis technique, insertion into the gene of interest is selected for by crossing an individual homozygous for the earlier identified recessive mutation with a wild-type individual having an autonomous transposon (alternatively, an individual carrying both a stable transposase source and the mutation of interest is crossed with a wild-type individual containing a non autonomous transposon). The next generation, the M1, is screened for all the individuals showing the mutant 
phenotype. These individuals contain the original mutation as well as a new transposoninduced allele of the same gene.

The second type of directed mutagenesis experiment is used in schemes where the transposon moves preferentially to linked sites on the chromosome. A mapped TE that is linked to the gene of interest is mobilized. Insertions into the specified gene are identified in the M1, or the linked transposon is allowed to mobilize in a wild-type background, followed by screening of M2 for mutations.

In either case, a biased preference for transposition to linked sites leads to increase in the frequency of mutations induced in the specific gene, relative to a random tagging procedure.

\section{Enhancer and gene traps}

Enhancer traps are transposons developed for identifying genes on the basis of their expression behavior. The transposon has a reporter gene, controlled by a weak or minimal promoter. When transposon is inserted near an enhancer, it causes the reporter gene to be expressed in a waygoverned by that enhancer. This increases the possibility to identify genes having tissuespecific or other regulated patterns. Enhancertrap screens identify genes not usually observed in regular tagging screens, such as those genes, having no visible phenotype when disrupted. They can also be aided in suggesting new roles for genes. For example, nearby insertion of an enhancer trap could exhibit interesting adult expression patterns in a gene that, when disrupted becomes lethal in an embryo. Enhancer-trap lines yield useful tissue, cell-type, and developmentally specific markers that can be analyzed in diverse mutant backgrounds under different conditions. Enhancer-trap screens are effectively used to identify various gene expression patterns in different organisms, like Arabidopsis and Drosophila.

Similarity exists between gene traps and enhancer traps but the transposon used here lacks promoter and has reporter whose expression requires the transposon to be inserted downstream of the promoter of the expressed gene. RNA splicing-acceptor sequences are introduced upstream of the coding region of the reporter gene to permit fusion of the reporter gene to the tagged gene if the transposon inserts into an intron.

Cloning genes identified by a gene-trap transposon is often easier than cloning enhancer-trapped genes, because reporter gene expression needs gene traps to be inserted downstream of the promoter. Enhancers can be inserted at a distance from gene being regulated by it. Chromosome walking, from the site of transposon insertion is sometimes needed to clone the endogenous enhancer-regulated gene.

\section{Cloning tagged genes: from insertion to sequence}

A marker in the form of transposon linked with the mutation is required to be identified to proceed with the cloning. This is done by Southern blotting DNA from progeny obtained from the putatively tagged mutant. The probe used here is transposon specific that identifies homozygous mutant progeny and is absent in wild-type progeny that do not segregate for the mutant phenotype.

Sometimes many transposons segregate in the background, so finding a transposon linked to the mutant phenotype is difficult. Either a large number of progeny needs to be examined or strain transposons to segregate background transposons. Once a linked transposon is identified, there are a number of 
different ways to clone the gene in which a transposon has inserted. Three commonly used methods are described here.

\section{Plasmid rescue}

The main requirement for the use of this method is that the transposon used for tagging should contain a part of a selectable plasmid (e.g., pBR322) at one end of the transposon, plasmid rescue is used to isolate a fragment of flanking host DNA. Genomic DNA from the tagged individual is digested with a restriction enzyme that releases the selectable plasmid from the transposon but does not cut within the plasmid itself. This creates a linear piece of DNA containing the plasmid sequence and a small fragment of flanking host DNA. The products of the digestion are re-ligated at a higher dilution to ensure intramolecular ligation products. The ligation products are transformed into E. coli, and then the cloned flanking DNA is isolated.

\section{Inverse polymerase chain reaction (IPCR)}

IPCR is another method for isolating host sequences flanking a transposon insertion. PCR using two transposon-specific oligonucleotide primers, each reading outward from the ends of the transposon sequence into the flanking DNA, are used to amplify the flanking host DNA. Then the amplified product is cloned.

\section{Library screening}

A third option is to make a library using genomic DNA from the tagged mutant that has been digested with an enzyme that does not cut within the inserted transposon. The recombinant vector containing the transposon also contains some flanking host DNA. A transposon-specific probe is used to screen the library and identify clones containing the transposon and the flanking DNA.

\section{Confirmation}

After cloning of the DNA with host region flanking, it should be used to probe Southern blots of DNA extracted from homozygous mutant and wild-type plants to look for a band difference, indicating transposon insertion into the complementary sequences in the mutant.

This is done to prove that the cloned DNA actually represents the host DNA flanking the transposon and is not an artifact of cloning. Then the cloned flanking sequences are sequenced directly or used as probes to obtain full-length sequences for further analysis. Chromosome walking is needed to identify the gene of interest, in case, the insertion of transposon is not into coding sequence of the disrupted gene itself. (or in the case of an enhancer trap, if the transposon is not in a gene).

Transforming the cloned gene back into the mutant and then rescuing the mutant phenotype is the most convincing way to prove that a mutant phenotype results from transposon disruption of the cloned gene. It is also possible to isolate and analyze different alleles of the gene from independently derived mutants. If all of the mutant alleles harbor mutations in the cloned gene, it is good evidence that the correct gene has been identified.

Phenotypic revertants that show a loss of the transposon from the cloned sequence, when they are examined by sequencing or Southern blotting techniques, also confirm the identity of the tagged gene. In the case of enhancertrapped genes, where there is no mutant phenotype associated with the insertion, expression analysis of the cloned gene should closely mimic that of the enhancer-trap reporter gene. 
Transposon tagging strategies: from gene to mutant

\section{Site-selected mutagenesis}

Gene of known sequence is being inserted with transposon and causes its mutation leading to its null expression, which helps in identifying the function of the gene. Homology study and expression pattern analysis are often used for the same purpose.

To obtain a site-selected mutation in a gene of interest, a large population of transposants is generated and divided into pools. Two types of primers are needed for the PCR reaction; primers specific to the gene of interest and transposon-specific primers, one reading "out" from each end of the transposon into the host DNA flanking the insertion. PCR is performed using one gene-specific primer, one transposon-specific primer, and DNA obtained from a pool of transposants.

If an individual has an insertion in or near the gene of interest, the gene-specific primer and the transposon-specific primer are positioned so that a PCR product is amplified. Nested primers are often used to ensure specificity of the amplified band. Then pools of transposants from which a product is amplified are subdivided and rescreened until a single individual harboring the insert is obtained.

Maintenance of viable siblings of the original transposants is needed for further studies as the original individuals are often dead by the time tagged individuals are identified. In the case of plants, this is simple because seeds from the transposants can be maintained for reasonable periods of time.

In some cases, the original insertion does not change gene function sufficiently to create a visible phenotype. Then, it becomes important to remobilize the transposon and select for excision phenomenon that fully disrupt the gene.

It is concluded that the transposon tagging techniques are valuable and important for several reasons. They are aided to identify and clone various genes having distinguished phenotypes, and enhancer-trap and gene-trap systems are used for screening genes based on their expression patterns.

Assessing a tagged allele of a gene is more often a shortcut to cloning when compared to traditional methods, such as chromosome walking. Now, creation of site-selected tagging screens helps identifying transposon insertions into genes of known sequence. These insertions result in null alleles or are used to produce null alleles of genes for which no earlier mutation is known. Null alleles are extremely useful to know the functional roles of the gene sequence under study. They are utilized in genetic studies, for example, in analyzing double-mutant interactions.

Transposon-induced alleles are required to produce an allelic series by remobilization of the transposon and selection for reinsertion elsewhere in the gene or coding sequence. Multiple alleles of a gene can give insights into its function.

Somatic excision occurrences are used to study the role of a gene in different tissues at different situations during development. For all the different reasons, transposon tagging is and will retain to be a useful tool for gene cloning and gene analysis.

\section{References}

Allen SE, Nowacki M (2017) Necessity is the mother of invention: ciliates, transposons, and transgenerational 
inheritance. Trends Genet. 33:197-207.

Arkhipova IR (2017) Using bioinformatic and phylogenetic approaches to classify transposable elements and understand their complex evolutionary histories. Mob DNA. 0103-2.

Athma, P., E. Grotewold and T. Peterson, (1992) Insertionalmuta genesis of the maize $\mathrm{P}$ gene by intragenic transposition of Ac. Genetics 131: 199-209.

Bailey, JA, et al., (2000) Molecular evidence for a relationship between LINE-1 elements and $\mathrm{X}$ chromosome inactivation: The Lyon repeat hypothesis. Proceedings of the National Academy of Sciences 97, 6634-6639.

Barsh, G. S., Seeburg, R H. and Gelinas, R. E. (1983) Nucleic Acid,; Res. 11, 3939-3958

Berg, D. and Howe, M.M. (eds) Mobile DNA, American Society tbr Microbiology (in press)

Boeke JD, Garfinkel DJ, Styles CA, Fink GR (1985) Ty elements transpose through an RNA intermediate Cell, 40:491-500.

Brown PO, Bowerman B, Varmus HE, Bishop JM (1987) Correct integration of retroviral DNA in vitro. Cell,49:347-356.

Chen, J., Greenblatt, I. M. and Dellaporta. S. L. (1987) Genetics 117, 109-116

Cheung S, Manhas S, Measday V (2018) Retrotransposon targeting to RNA polymerase III-transcribed genes. Mob DNA, 9:14.

David A. Jones, Colwyn M. Thomas, Kim E. Hammond-Kosack, Peter J. BalintKurti, Jonathan D. G. Jones (1994). Isolation of the Tomato Cf-9 Gene for Resistance to Cladosporiumfulvum by Transposon Tagging. Science 266: 789792.

Dobzhansky T (1964) Genetics- and the Orl o, in of Species (3rd edn), Columbia University Press.

Doring HP and Starlinger P (1986) Annu. Rev. Genet. 20, 175-200.

Engels WR and Preston CR (1984) Genetics
107,6574478

Errede B, Company $\mathrm{M}$ and Hutchison CA (1987) Mol. Cell. Biol. 7, 258-264.

Fanning TG and Singer MF (1987) Biochim. Biophys. Acta 910, 203-212.

Feschotte C, et al., (2002)Plant transposable elements: Where genetics meets genomics. Nature Reviews Genetics 3, 329-341.

Finnegan DJ and Fawcett DH (1986) Transposable Elements in Drosophila melanogaster (Oxford Surveys of Euka ,otic Genes, Vol. 3), (Maclean, N., ed.), pp 1 2, Oxford University Press.

Geyer PK, Green, MM and Cortes VG (1988) Proc. Natl Acad. Sci. USA 85, 859343597.

Gotea V, Makalowski W (2006) Do transposable elements really contribute to proteomes? Trends Genet; 22:260-267.

Horváth V, Merenciano M, González J. Revisiting the relationship between transposable elements and the eukaryotic stress response. Trends Genet; 33:832-841.

Izsvak Z and Ivics Z (2004) Transposable elements for transgenesis and insertional mutagenesis in vertebrates. Mobile Genetic Elements, Springer; 255-276.

Kazazian HH (2004) Mobile elements: Drivers of genome evolution. Science 303, 1626-1632.

Kazazian HH and Moran JV (1998) The impact of L1 retrotransposons on the human genome. Nature Genetics 19, $19-24$

Kazazian HH, et al., (1998) HaemophiliaA resulting from de novo insertion of $\mathrm{L} 1$ sequences represents a novel mechanism for mutation in man. Nature 332, 164-166.

Rubin GM, Kidwell MG, Bingham PM: The molecular basis of P-M hybrid dysgenesis: the nature of induced 
mutations. Cell. 1982, 29: 987-994.

Kim, H-Y. et al., (1987) Proc. Nall Acad. Aci. USA 84, 5863-5867.

Kingsman AJ and Kingsman S (1988) @1153, 333-335.

Kingsman AJ, Chater KF and Kingsman SM (eds) (1988) Transposition, Cambridge University Press.

Kofler R, Nolte V, Schlötterer C (2015) Tempo and mode of transposable element activity in Drosophila. PLOS Genet. 2015;11:e1005406.

Koga A, et al., (2006) Vertebrate DNA transposon as a natural mutator: The medaka fish Tol2 element contributes to genetic variation without recognizable traces. Molecular Biology and Evolution 23, 1414-1419.

Lanciano S, Mirouze M (2018) Transposable elements: all mobile, all different, some stress responsive, some adaptive? CurrOpin Genet Dev. 2018;49:106-114. Langley CH et al., (1988) Genet. Res. 52, 223-235.

Lazarow K, Doll M-L, Kunze R (2013) Molecular Biology of Maize Ac/Ds elements: an overview. In : Peterson T, editor. Plant transposable elements. Totowa : Humana Press; pp. 59-82.

Mackay TFC (1985) Genetic; 111, 351-374.

Maeda N and Smithies O (1986) Annu. Rev. Genet. 20, 81-108.

Makarevitch I, Waters AJ, West PT, Stitzer $\mathrm{M}$, Hirsch CN, Ross-Ibarra J, et al., Transposable elements contribute to activation of maize genes in response to abiotic stress. PLoS Genet. 2015; 11:e1004915.

McCarrey JR and Thomas K (1987) Nature 326, 501-504.

McClintock B (1951) Mutable loci in maize. Carnegie Institution of Washington Yearbook 50, 174-181.

McLean, P. McClintock and the Ac/Ds transposable elements of corn, www.ndsu.nodak.edu/instruct/mcclean/ plsc431/transelem/trans1.htm (1997).

Miki Y, et al., (1992) Disruption of the APC gene by a retrotransposal insertion of $L 1$ sequence in colon cancer. Cancer Research 52, 643-645.

Miura A et al., (2001) Mobilization of transposons by a mutation abolishing full DNA methylation in Arabidopsis. Nature 411, 212-214.

Montgome D' E, Charlesworth B and Langley CH (1987) Genet. Res. 49, 31 1.

Moran, J. V., et al., (1999) Exon shuffling by L1 retrotransposition. Science 283, 1530-1534.

Ohno S. (1972) So much "junk" DNA in our genome. In: Smith $\mathrm{HH}$, editor. Evolution of genetic systems. Brookhaven: SympBiol; pp. 366-370.

Parkhurst SM et al., (1988) Genes Dev. 2, 1205-1215.

Peterson T, (1990) Intragenic transposition of Ac generates a new allele of the maize $P$ gene. Genetics 126: 469-476.

Roeder GS and Fink GR (1983) in Mobile Genetic Elements (Shapiro, J., ed.) pp. 299-328, Academic Press.

Rozenzweig B, Liao LW, Hirsh D (1983) Sequence of the C. elegans transposable element Tc1. Nucleic Acids Res. 11(12): 4201-9.

SanMiguel P, et al., (1996) Nested retrotransposons in the intergenic regions of the maize genome. Science 274, 765-768.

Schnable PS, Ware D, Fulton RS, Stein JC, Wei F, Pasternak S, et al., The B73 maize genome: complexity, diversity, and dynamics. Science. 2009; 326:1112-1115.

Schneuwly ,Kt, roiwa, A and Gehring WJ (1987) EMBOJ. 6, 201-206.

Schwarz-Sommer Z et al., (1985) EMBOJ. 4, 591-597.

Slotkin RK and Martienssen R (2007) Transposable elements and the epigenetic regulation of the genome. 
Nature Reviews Genetics 8, 272-285.

Stein JE et al., (1983) Proc. Natl Acad. ScL USA 80, 6485-6489.

Wayne P Fitzmauric, Long V Nguyen, Earl A

Wernsman, William $\mathrm{F}$ Thompson and Mark A Conkling (1999) Transposon Tagging of the Sulfur Gene of Tobacco Using Engineered Maize Ac/Ds Elements. Genetics Society of America 153: 1919-1928.

Wessler SR, Baran G and Varagona M (1987) Science 237, 916-918.

Whitham S, Dinesh Kumar SP, Choi D, Hehl

R, Corr C, Baker B (1994) The product of tobacco mosaic virus resistance gene $\mathrm{N}$ : similarity to toll and interleukin-1 receptor. Cell; 23; 78(6): 1101-15.

Wicker T, Sabot F, Hua-Van A, Bennetzen JL, Capy P, Chalhoub B, et al., (2007) A unified classification system for eukaryotic transposable elements. Nat Rev Genet. 2007; 8:973-982.

Yang N and Kazazian HH (2006) L1 retrotransposition is suppressed by endogenously encoded small interfering RNAs in human cultured cells. Nature Structural and Molecular Biology 13, 763-771.

\section{How to cite this article:}

Sadia Perween, Deepak Kumar and Anand Kumar. 2020. A Review on Transposons and its Utilization as Genetic Tool. Int.J.Curr.Microbiol.App.Sci. 9(02): 1874-1884. doi: https://doi.org/10.20546/ijcmas.2020.902.214 